\title{
A new treatment for neurogenic inflammation caused by EV71 with CR2-targeted complement inhibitor
}

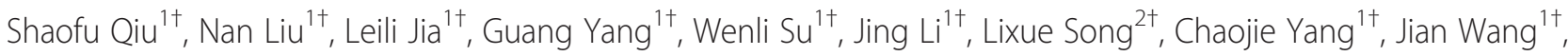
Chuanfu Zhang ${ }^{1}$, Zhongqiang Wang ${ }^{1}$, Fei Qiao ${ }^{3}$, Stephen Tomlinson ${ }^{3}$, Carl Atkinson ${ }^{3}$, Yansong Sun ${ }^{1}$, Liuyu Huang ${ }^{1}$, Hongbin Song ${ }^{1^{*}}$, Yong Wang ${ }^{1 *}$ and Zhenjun $\mathrm{Li}^{4^{*}}$

\begin{abstract}
Background: Enterovirus 71 (EV71), one of the most important neurotropic EVs, has caused death and long-term neurological sequelae in hundreds of thousands of young children in the Asia-Pacific region in the past decade. The neurological diseases are attributed to infection by EV71 inducing an extensive peripheral and central nervous system (CNS) inflammatory response with abnormal cytokine production and lymphocyte depletion induced by EV71 infection. In the absence of specific antiviral agents or vaccines, an effective immunosuppressive strategy would be valuable to alleviate the severity of the local inflammation induced by EV71 infection.
\end{abstract}

Presentation of the hypothesis: The complement system plays a pivotal role in the inflammatory response. Inappropriate or excessive activation of the complement system results in a severe inflammatory reaction or numerous pathological injuries. Previous studies have revealed that EV71 infection can induce complement activation and an inflammatory response of the CNS. CR2-targeted complement inhibition has been proved to be a potential therapeutic strategy for many diseases, such as influenza virus-induced lung tissue injury, postischemic cerebral injury and spinal cord injury. In this paper, a mouse model is proposed to test whether a recombinant fusion protein consisting of CR2 and a region of Crry (CR2-Crry) is able to specifically inhibit the local complement activation induced by EV71 infection, and to observe whether this treatment strategy can alleviate or even cure the neurogenic inflammation.

Testing the hypothesis: CR2-Crry is expressed in CHO cells, and its biological activity is determined by complement inhibition assays. 7-day-old ICR mice are inoculated intracranially with EV71 to duplicate the neurological symptoms. The mice are then divided into two groups, in one of which the mice are treated with CR2-Crry targeted complement inhibitor, and in the other with phosphate-buffered saline. A group of mice deficient in complement C3, the breakdown products of which bind to CR2, are also infected with EV71 virus. The potential bioavailability and efficacy of the targeted complement inhibitor are evaluated by histology, immunofluorescence staining and radiolabeling.

Implications of the hypothesis: CR2-Crry-mediated targeting complement inhibition will alleviate the local inflammation and provide an effective treatment for the severe neurological diseases associated with EV71 infection.

\footnotetext{
*Correspondence: hongbinsong@263.net; ywang7508@yahoo.com.cn; lizhenjun@icdc.cn

${ }^{\dagger}$ Equal contributors

${ }^{1}$ Institute of Disease Control and Prevention, Academy of Military Medical

Sciences, Beijing 100071, China

Full list of author information is available at the end of the article
} 


\section{Background}

Enterovirus 71 (EV71) is the major causative agent of hand-foot-and-mouth disease (HFMD) [1]. Since the virtual eradication of the poliovirus, EV71 has been recognized as the most important neurotropic EV. It can cause various neurological diseases, such as aseptic meningitis, acute flaccid paralysis, brainstem encephalitis and fatal neurogenic pulmonary edema [2,3].

Since the first identification of EV71 in 1969, several epidemic outbreaks have been reported in the AsiaPacific region (Malaysia in 1997, Australia in 1999, Singapore in 2000, Japan in 1997 and 2000, Taiwan in 1998, 2000, 2001 and 2002, and Mainland China in 1998, 2004 and 2008) [3-6]. There were more than 1.1 million HFMD cases including 353 deaths due to the neurological disease in China in 2009 [7]. Mortality was particularly high in EV71-induced brainstem encephalitis complicated with pulmonary edema, especially in children under 5 years of age. EV71 infection has therefore become an important public health problem in the world, particularly in the Asia-Pacific region. EV71 displayed genetic diversity and the virus circulating in this region underwent rapid evolutionary change [8,9], which hampered the development of antiviral agents and vaccines for EV71 infection. As currently no specific antiviral agents or vaccines are available, we should seek a new therapeutic approach to alleviate the severity of EV71-induced neurological diseases.

\section{Presentation of the hypothesis}

\section{EV71 is involved in the inflammatory response of the} central nervous system

In recent EV71 epidemics in the Asia-Pacific region, the serious complications were mainly associated with the central nervous system (CNS), and the primary lethal symptom was neurogenic pulmonary edema [10]. Magnatic Resonance Imaging and autopsy examinations showed that the pathological lesions occurred predominantly in the brainstem and the spinal cord, rather than in the lung or heart $[1,11]$. The EV71-associated inflammatory response was found mainly in the CNS region but not in other organs of EV71-infected patients $[2,3,12]$, indicating that the CNS is the major target of EV71 infection. EV71 can enter the CNS through peripheral nerves via retrograde axonal neuronal transmission way or via viremic spread through the blood-brain barrier (BBB). It then induces the human immune cell lines and triggers NF-кь activation to produce proinflammatory cytokines leading to an inflammatory response of the CNS $[2,3,10]$. Besides, many molecules, such as cyclooxygenase- 2 and its metabolite, the cellular protein Cdk5 and others, can facilitate EV71 replication in neural cells and induce neural apoptotic cell death [3]. It is now widely accepted that the extensive peripheral and CNS inflammatory response accompanied by the excessive release of cytokines and chemokines is responsible for the pathogenesis of EV71-associated neurological diseases. These can cause neuronal degeneration, CNS necrosis and destruction of vasomotor function in the brainstem, leading to autonomic nervous system dysregulation and even fatal neurogenic pulmonary edema [13-16]. Patients with brainstem encephalitis and neurogenic pulmonary edema showed elevated levels of inflammatory CNS cytokines, including TNF- $\alpha$, IL- $1 \beta$, and IL-6, IL-10, IL-13 and IFN- $\gamma$, and a marked depletion of CD4 $4^{+}$ and $\mathrm{CD}^{+} \mathrm{T}$ cells and NK cells $[1,11,14]$, demonstrating the correlation between the extensive CNS inflammatory response and EV71-associated neurological diseases.

\section{Inflammatory injury induced by invading pathogens is associated with complement activation}

Complement is a key system for immune surveillance and homeostasis, and it bridges the innate and acquired immune responses $[17,18]$. Under normal circumstances, the immune response recognizes, attacks and eliminates the invading pathogens and this response is beneficial for the host. However, inappropriate or excessive activation of the complement system can result in severe inflammation or tissue injury involved in numerous pathological conditions. Previous studies have noted that many diseases, including the neurological diseases known as transmissible spongiform encephalopathies (TSEs), are due to inflammatory injury. This injury occurs as a result of immune activation by the invading pathogens, instead of being directly induced by the pathogens themselves. TSEs are fatal, neurodegenerative diseases caused by prion protein $\mathrm{PrP}^{\mathrm{Sc}}$. $\mathrm{PrP}^{\mathrm{Sc}}$ present in the brain can activate complement, and the deposited complement components $\mathrm{C} 1 \mathrm{q}, \mathrm{C} 3 \mathrm{~b}$ and the membraneattack complex have all been detected in the brains of patients with prion disease, the pathology of which is due to the propagation of inflammation [19]. It has been proved that an excessive inflammatory reaction in lung tissues induced by influenza virus infection is closely related to complement activation, and complement activation can seriously influence the severity of lung injury $[18,20]$.

The detailed pathogenesis of enteroviruses including EV71 remains unclear, but it has been documented that enterovirus infection can induce an extensive peripheral and CNS inflammatory response $[3,11,14]$. Our previous results have proved that the complement components C1q and C3 are observed as obvious depositions in the brainstems of mice showing the neurological symptoms of EV71 infection (data not shown), indicating that the inflammatory response in the mouse CNS is associated with complement activation. Anderson et al. showed that coxsackievirus B3 (CVB3) capsid proteins could interact with complement $\mathrm{C} 3$ and activate the alternative 
complement pathway after in vivo murine infection [21]. The research conducted by Shih et al. revealed that EV71 infection could lead to an increased level of mRNAs encoding complement proteins [22]. These results also illustrated the activation of complement due to EV71 infection.

\section{Targeted complement inhibition is a potential therapeutic strategy for the inflammatory response}

Complement activation can generate inflammatory products including C2a, C3a, C4a and C5a which mediate tissue injury [18,23]. C3a and C5a have the functions of anaphylatoxins and chemoattractants with a wide range of bioactive properties which can induce and increase the inflammatory reaction $[24,25]$. Complement proteins on cell membranes can be receptors for activated complement proteins or proteins that regulate complement. Complement receptor 2 (CR2), a member of the C3 binding protein family, serves as an important interface between the complement system and adaptive immunity. The specific ligands for CR2 are iC3b, C3dg and C3d, all being cellbound breakdown fragments of $\mathrm{C} 3$ which are deposited on the complement-activating cell surface $[24,26,27]$. Thus, CR2 is a rational target for the delivery of complement inhibitors such as Crry and CD59 to inflammatory sites that have been induced by complement activation. As Crry has different levels of species selectivity and acts as an important complement regulatory protein, it is considered as an effective complement inhibitor which can regulate complement activation by inhibiting the activity of $\mathrm{C} 3 / \mathrm{C} 5$ convertase. CR2-Crry, an ideal targeted inhibitor which has shown increased bioavailability and efficacy and decreased immunosuppression $[18,27,28]$, could provide an appropriate therapeutic strategy for many inflammation-related diseases such as lung tissue injury induced by influenza virus, postischemic cerebral injury and spinal cord injury.

\section{Hypothesis}

Previous studies indicated that CR2-Crry had an appropriate bioavailability for targeted complement inhibition for the treatment of spinal cord injury and cerebral ischemia/ reperfusion injury $[27,28]$. Our experiments also proved that CR2-Crry could obviously inhibit the pulmonary inflammatory response caused by influenza virus [18]. Thus, targeted complement inhibition induced by CR2-Crry may be an effective therapeutic strategy for EV71 infection of the CNS. Complement activation is a double-edged sword leading to both physiological defense and pathological damage, and systematic complement inhibition may result in potential side effects including infection [25]. Thus, a new treatment strategy for EV71 infection is required. Here, the complement inhibitor CR2-Crry, which targets the sites of complement activation, is proposed as a specific inhibitor of local complement activation without causing severe systemic side effects.

\section{Testing the hypothesis}

The recombinant fusion protein CR2-Crry is prepared by joining the mouse CR2 sequence to the sequence encoding the extracellular region of mouse Crry, and is expressed and purified as described previously [26,29]. The biological activity of the recombinant CR2-Crry is characterized by complement inhibition assays. 7-day-old ICR mice are inoculated intracranially with EV71 $\mathrm{BrCr}$ strain to duplicate the neurological symptoms. Then the mice are divided into two groups, in one of which the mice are treated with CR2-Crry, and in the other with phosphate-buffered saline. A group of mice deficient in complement $\mathrm{C} 3$ are also each infected with EV71 virus. Animals are perfused transcardially with isotonic saline followed by $4 \%$ paraformaldehyde. The brains and spinal cords are either frozen for cryosectioning or processed in paraffin. Immunofluorescence staining is used to assess the presence of complement component C3 and CR2Crry in the sites of inflammatory, and radiolabeling is conducted to detect the tissue distribution of ${ }^{125}$ I-labeled CR2-Crry in control mice and in mice infected with EV71.

\section{Implications of the hypothesis}

Since the outbreak of HFMD in Shandong and Anhui provinces in 2008, EV71 is gradually spreading to other provinces of China and has become a serious threat to public health in these regions. The EV71-associated neurological diseases which often cause deaths in children under 5 years of age are not directly induced by the virus itself, but are attributable to the inflammation due to EV71 infection. Whilst antiviral therapy is one possible approach, it is perhaps more important to inhibit the inflammatory response caused by EV71. Compared with systemic inhibition, CR2-mediated targeting complement inhibition should greatly reduce the inflammatory reaction or tissue injury resulting from excess complement activation. Thus, verification of the current hypothesis could lead to an effective treatment for EV71 infection.

\section{Competing interests}

The authors declare that they have no competing interests.

\section{Authors' contributions}

SFQ, NL and LLJ prepared the manuscript. SFQ, NL, LLJ, GY, WLS, LJ, LXS, CJY, JW, CFZ, ZQW, FQ, ST, CA, YSS, LYH, HBS, YW and ZJL participated in developing the hypothesis and collaborated in writing and reviewing of the article. All authors read and approved the final manuscript.

\section{Acknowledgements}

This work was funded by the Mega-projects of Science and Technology Research of China (No. 2012ZX10004215 and 2011ZX10004-001) and the National Natural Science Foundation of China (No. 81171554).

Author details

${ }^{1}$ Institute of Disease Control and Prevention, Academy of Military Medical Sciences, Beijing 100071, China. ${ }^{2}$ Department of Pharmacology, General Hospital of Beijing Military Region, Beijing 100700, China. ${ }^{3}$ Department of Microbiology and Immunology, Medical University of South Carolina, Charleston, South Carolina 29425, USA. ${ }^{4}$ State Key Laboratory for Infectious 
Disease Prevention and Control, National Institute for Communicable Diseases Prevention and Control, Chinese Center for Disease Control and Prevention, Beijing 102206, China.

Received: 29 April 2011 Accepted: 2 November 2012

Published: 23 November 2012

\section{References}

1. Wang SM, Liu CC: Enterovirus 71: epidemiology, pathogenesis and management. Expert Rev Anti Infect Ther 2009, 7:735-742.

2. Chen CS, Yao YC, Lin SC, Lee YP, Wang YF, Wang JR, Liu CC, Lei HY, Yu CK: Retrograde axonal transport: a major transmission route of enterovirus 71 in mice. J Virol 2007, 81:8996-9003.

3. Weng KF, Chen LL, Huang PN, Shih SR: Neural pathogenesis of enterovirus 71 infection. Microbes Infect 2010, 12:505-510

4. Lee MS, Chang LY: Development of enterovirus 71 vaccines. Expert Rev Vaccines 2010, 9:149-156

5. Vallet S, Legrand Quillien MC, Dailland T, Podeur G, Gouriou S, Schuffenecker I, Payan C, Marcorelles P: Fatal case of enterovirus 71 infection, France, 2007. Emerg Infect Dis 2009, 15:1837-1840.

6. Yu H, Chen W, Chang H, Tang R, Zhao J, Gan L, Liu B, Chen J, Wang M: Genetic analysis of the VP1 region of enterovirus 71 reveals the emergence of genotype A in central China in 2008. Virus Genes 2010, 41:1-4.

7. Han JF, Cao RY, Jiang T, Yu M, Liu W, Tian X, Qin ED, Cao WC, Qin CF: Echovirus 30 in EV71-associated hand, foot and mouth disease outbreak, Guangxi, China. J Clin Virol 2011, 50:348-349.

8. Bible JM, Pantelidis P. Chan PK, Tong CY: Genetic evolution of enterovirus 71: epidemiological and pathological implications. Rev Med Virol 2007, 17:371-379.

9. McMinn PC: An overview of the evolution of enterovirus 71 and its clinical and public health significance. FEMS Microbiol Rev 2002, 26:91-107.

10. Jia CS, Liu JN, Li WB, Ma CM, Lin SZ, Hao Y, Gao XZ, Liu XL, Xu YF, Zhang LF, Qin C: The cross-reactivity of the enterovirus 71 to human brain tissue and identification of the cross-reactivity related fragments. Virol J 2010, 7:47.

11. Wang SM, Lei HY, Huang KJ, Wu JM, Wang JR, Yu CK, Su IJ, Liu CC Pathogenesis of enterovirus 71 brainstem encephalitis in pediatric patients: roles of cytokines and cellular immune activation in patients with pulmonary edema. J Infect Dis 2003, 188:564-570.

12. Lee TC, Guo HR, Su HJ, Yang YC, Chang HL, Chen KT: Diseases caused by enterovirus 71 infection. Pediatr Infect Dis J 2009, 28:904-910.

13. Lin TY, Chang LY, Huang YC, Hsu KH, Chiu CH, Yang KD: Different proinflammatory reactions in fatal and non-fatal enterovirus 71 infections: implications for early recognition and therapy. Acta Paediatr 2002, 91:632-635.

14. Lin TY, Hsia SH, Huang YC, Wu CT, Chang LY: Proinflammatory cytokine reactions in enterovirus 71 infections of the central nervous system. Clin Infect Dis 2003, 36:269-274.

15. Wang SM, Lei HY, Su LY, Wu JM, Yu CK, Wang JR, Liu CC: Cerebrospinal fluid cytokines in enterovirus 71 brain stem encephalitis and echovirus meningitis infections of varying severity. Clin Microbiol Infect 2007, 13:677-682.

16. Wang SM, Lei HY, Yu CK, Wang JR, Su IJ, Liu CC: Acute chemokine response in the blood and cerebrospinal fluid of children with enterovirus 71-associated brainstem encephalitis. J Infect Dis 2008, 198:1002-1006.

17. Ricklin D, Hajishengallis G, Yang K, Lambris JD: Complement: a key system for immune surveillance and homeostasis. Nat Immunol 2010, 11:785-797.

18. Zhang $C, X u Y$, Jia L, Yang $Y$, Wang $Y$, Sun $Y$, Huang L, Qiao F, Tomlinson $S$, Liu $X$, et al: A new therapeutic strategy for lung tissue injury induced by influenza with CR2 targeting complement inhibitor. Virol J 2010, 7:30.

19. Sjoberg AP, Trouw LA, Blom AM: Complement activation and inhibition: a delicate balance. Trends Immunol 2009, 30:83-90.

20. Kopf M, Abel B, Gallimore A, Carroll M, Bachmann MF: Complement component $\mathrm{C} 3$ promotes T-cell priming and lung migration to control acute influenza virus infection. Nat Med 2002, 8:373-378.

21. Anderson DR, Carthy CM, Wilson JE, Yang D, Devine DV, McManus BM: Complement component 3 interactions with coxsackievirus B3 capsid proteins: innate immunity and the rapid formation of splenic antiviral germinal centers. J Virol 1997, 71:8841-8845.
22. Shih SR, Stollar V, Lin JY, Chang SC, Chen GW, Li ML: Identification of genes involved in the host response to enterovirus 71 infection. J Neurovirol 2004, 10:293-304.

23. Walport MJ: Complement. First of two parts. N Engl J Med 2001, 344:1058-1066.

24. Song H, He C, Knaak C, Guthridge JM, Holers VM, Tomlinson S: Complement receptor 2-mediated targeting of complement inhibitors to sites of complement activation. J Clin Invest 2003, 111:1875-1885.

25. Walport MJ: Complement. Second of two parts. N Engl J Med 2001, 344:1140-1144.

26. Atkinson C, Song H, Lu B, Qiao F, Burns TA, Holers VM, Tsokos GC, Tomlinson S: Targeted complement inhibition by C3d recognition ameliorates tissue injury without apparent increase in susceptibility to infection. J Clin Invest 2005, 115:2444-2453.

27. Qiao F, Atkinson C, Song H, Pannu R, Singh I, Tomlinson S: Complement plays an important role in spinal cord injury and represents a therapeutic target for improving recovery following trauma. Am J Pathol 2006, 169:1039-1047.

28. Atkinson C, Zhu H, Qiao F, Varela JC, Yu J, Song H, Kindy MS, Tomlinson S: Complement-dependent P-selectin expression and injury following ischemic stroke. J Immunol 2006, 177:7266-7274.

29. Huang Y, Qiao F, Atkinson C, Holers VM, Tomlinson S: A novel targeted inhibitor of the alternative pathway of complement and its therapeutic application in ischemia/reperfusion injury. J Immuno/ 2008, 181:8068-8076.

doi:10.1186/1743-422X-9-285

Cite this article as: Qiu et al.: A new treatment for neurogenic inflammation caused by EV71 with CR2-targeted complement inhibitor. Virology Journal 2012 9:285.

\section{Submit your next manuscript to BioMed Central and take full advantage of:}

- Convenient online submission

- Thorough peer review

- No space constraints or color figure charges

- Immediate publication on acceptance

- Inclusion in PubMed, CAS, Scopus and Google Scholar

- Research which is freely available for redistribution 